\title{
Stress relaxation in rolled AZ31 magnesium alloy sheets
}

\author{
J. Balík, P. Lukáč* \\ Charles University, Faculty of Mathematics and Physics, Department of Physics of Materials, \\ Ke Karlovu 5, 12116 Prague 2, Czech Republic
}

Received 14 June 2015, received in revised form 21 July 2015, accepted 22 July 2015

\begin{abstract}
Stress relaxation tests were performed at room and higher temperatures to analyze the determination of the strain rate sensitivity of dislocation stress and the activation volume in Mg-3Al-1Zn alloy sheets. The tensile axis was parallel to the rolling direction. The activation volume estimated at room temperature decreases with increasing stress at the beginning of the relaxation. The relaxation duration of at least $30 \mathrm{~min}$ was used. At higher temperatures, the activation volume can be determined only from data at the beginning relaxation up to $300 \mathrm{~s}$. Stress relaxation tests at higher temperatures for at least $30 \mathrm{~min}$ indicate that recovery processes play an important role in relaxation.
\end{abstract}

K e y w o r d s: magnesium alloy, stress relaxation, dislocations

\section{Introduction}

In a stress relaxation test, a specimen is deformed at a strain rate to a certain strain (stress) level, then the machine is stopped, and the specimen is allowed to deform inelastically. Stress relaxation (SR) is the time-dependent decrease of stress in the specimen deformed to the predetermined stress. The applied stress $\sigma_{0}$ at the beginning of the relaxation decreases with time $t$ during the stress relaxation. A stress decrement $\Delta \sigma=\sigma_{0}-\sigma(t)$ can be determined; $\sigma(t)$ is the stress during relaxation at time $t$. The specimen can be again reloaded and strained to a higher stress, and the test can be repeated. The stress relaxation technique has been demonstrated to be a useful experimental method to evaluate activation parameters that indicate the thermally activated processes during deformation $[1-7]$.

In the literature, there are different equations describing stress relaxation curves, e.g. $[1,8]$. Dotsenko [9] has reviewed, in details, calculations of the experimental stress relaxation curves.

In the present work, we use the general equation of SR that can be expressed in the following form:

$$
\dot{\varepsilon}=-\kappa \dot{\sigma},
$$

where $\dot{\varepsilon}$ is the plastic strain rate (also called true strain rate); the derivate of the stress, $\dot{\sigma}$, is called the stress (relaxation) rate. The elastic compliance of the set including load cell and specimen $\kappa=K A(\ell-K A \sigma)$, where $\ell$ and $A$ are the current length and cross section area of the sample, respectively, and $K=\Delta x_{\text {elast }} / \Delta F$ $\left(\Delta F\right.$ and $\Delta x_{\text {elast }}$ are the load and elastic extension increments, respectively). In this paper, $\varepsilon$ and $\dot{\varepsilon}$ are called plastic strain and plastic strain rate, respectively. The plastic strain rate during SR can be determined by measuring the slope of the relaxation curve, i.e. the stress rate, and using Eq. (1). It is worthy to mention that the plastic strain rate during SR changes (decreases) over a wide range.

Stress relaxation tests are often used for estimating the activation volume $V=b d L$, where $b$ is the magnitude of the Burgers vector, $d$ is the activation distance, and $L$ is the mean length of dislocation segments between short-range obstacles. The value of the activation volume helps to identify the thermally activated mechanism. A single thermally activated process can be described by the Arrhenius equation in the form $[10]$ :

$$
\begin{aligned}
& \dot{\gamma}=\dot{\gamma}_{0} \exp [-\Delta G / k T], \\
& \dot{\varepsilon}=\dot{\varepsilon}_{0} \exp [-\Delta G / k T],
\end{aligned}
$$

where $\dot{\gamma}$ is the shear strain (deformation) rate, $\dot{\varepsilon}$ is

*Corresponding author: tel.: +420 951551 366; e-mail address: lukac@met.mff.cuni.cz 
the plastic strain rate, $\dot{\gamma}_{0}$ and $\dot{\varepsilon}_{0}$ are pre-exponential factors containing the density of mobile dislocations, $k$ is the Boltzmann constant, and $T$ is the absolute temperature. The Gibbs free energy $\Delta G$ is a function of the shear stress, $\tau$, (Eq. (2a)), or the flow stress, $\sigma$ (Eq. (2b)). In a simple case

$$
\Delta G=\Delta G_{0}\left(1-\tau / \tau_{\mathrm{m}}\right),
$$

where $\Delta G_{0}$ is the activation energy at zero stress (it is also called the obstacle energy), and $\tau_{\mathrm{m}}$ is a maximum level of deformation resistance [11]. The activation volume is defined as the negative partial derivative of $\Delta G$ with respect to $\tau[10]$, i.e.

$$
V=-(\partial \Delta G / \partial \tau)=\Delta G_{0} / \tau_{\mathrm{m}}
$$

It should be mentioned that, in polycrystal deformation, the applied stress is related to the shear stress as $\sigma=m \tau$ (and $\varepsilon=\gamma / m$ ), where $m$ is the Taylor factor. In $\mathrm{Mg}$ alloys, $m$ value depends on the sample texture [12]. The stress relaxation curves are very often analyzed under the assumption that the dislocation structure does not change during the stress relaxation test. In other words: the effects of strain hardening/softening during the relaxation are neglected, and the internal (athermal) stress is assumed to be constant. It can be fulfilled at low temperatures where no recovery process occurs. Studies of the deformation behavior of magnesium alloys have demonstrated that recovery processes should be considered when the magnesium alloy is deformed at elevated temperatures [4]. In many cases, therefore, duration of the SR tests was chosen to about 300 s. Trojanová and Lukáč [4] have reported that the value of the internal (athermal) stress decreases very rapidly with increasing temperature.

The aim of the present work is to investigate the stress relaxation behavior of rolled magnesium alloy AZ31 sheets at room and elevated temperature to describe the effect of testing temperature on the behavior. Duration of the SR was chosen at least $30 \mathrm{~min}$.

\section{Experimental procedure}

The material used in this study was magnesium alloy AZ31 with composition Mg3Al-0.8Zn-0.2Mn (wt.\%). Specimens for experiments were processed from as-received rolled sheets of a thickness of $1.6 \mathrm{~mm}$. The resulting microstructure of specimens after annealing at $300^{\circ} \mathrm{C}$ for $8 \mathrm{~h}$ appears uniform, equiaxial with a mean grain size of $15 \mu \mathrm{m}$, and free of twins. The sample preparation details are described elsewhere [13]. Annealed samples display a basal texture that was determined using a Pananalytical Xpert MRD diffractometer and the Philips Xpert Texture software -

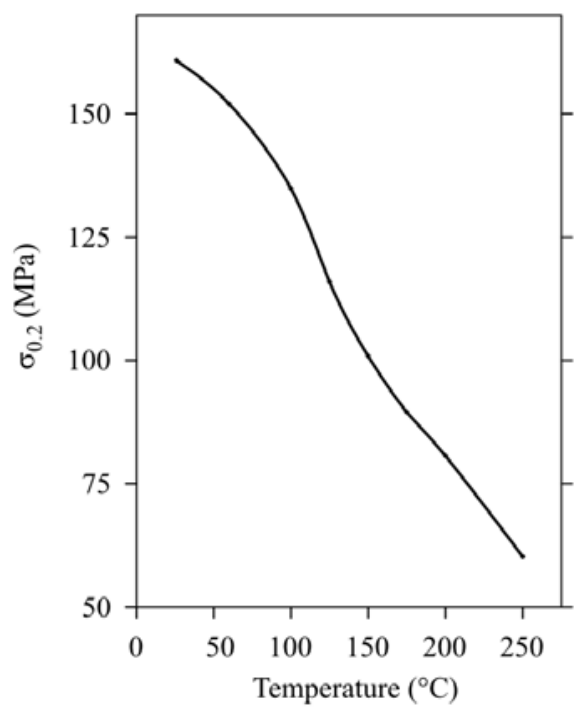

Fig. 1. Variation of the yield strength with temperature; strain rate $\dot{\varepsilon}=1.3 \times 10^{-3} \mathrm{~s}^{-1}$.

details and results have been reported by Balík et al. [14].

Tensile tests including stress relaxation tests of flat tension specimens with a gauge length of $25 \mathrm{~mm}$ and a cross section of $1.6 \times 6 \mathrm{~mm}^{2}$ were carried out on an Instron machine at different temperatures at an initial strain rate of $1.3 \times 10^{-3} \mathrm{~s}^{-1}$. The load axis was parallel to the rolling direction. The specimen was held for 1.5-2 $\mathrm{h}$ at the testing temperature before starting the test. The temperature in the furnace was controlled to within $\pm 0.5^{\circ} \mathrm{C}$. The stress drop during relaxation was recorded as a function of time with a sampling period of $0.1 \mathrm{~s}$.

\section{Experimental results}

The true stress-true strain curves of AZ31 sheets deformed in the rolling direction are very temperature sensitive. The deformation behavior is very similar as that described in details elsewhere $[5,13]$. The flow stress decreases and elongation to fracture increases with increasing temperature. The temperature dependence of the yield strength is shown in Fig. 1. Figure 2 shows two stress relaxation curves obtained at practically same strains at 23 and $125^{\circ} \mathrm{C}$. The effect of testing temperature can be seen on the SR curve. The difference between the stress at the beginning of $\mathrm{SR}$ and that at the relaxation end is temperature dependent. The stress drop is higher for a higher temperature.

Figure 3 shows the plots of the plastic strain rate calculated using Eq. (1) against the flow stress during SR for three different flow stresses at the beginning of $\mathrm{SR}$ at room temperature. The plots are also called kin- 


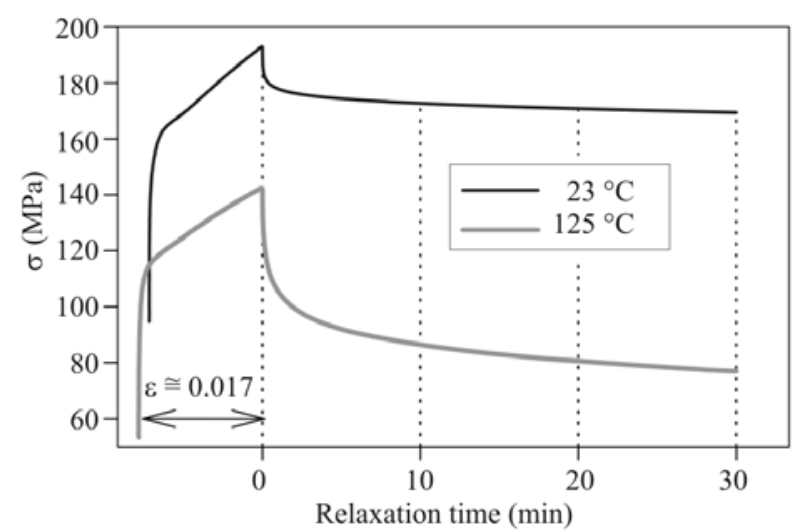

Fig. 2. Examples of relaxation curves; stress-time curves at the same strain at 25 and $125^{\circ} \mathrm{C}$.

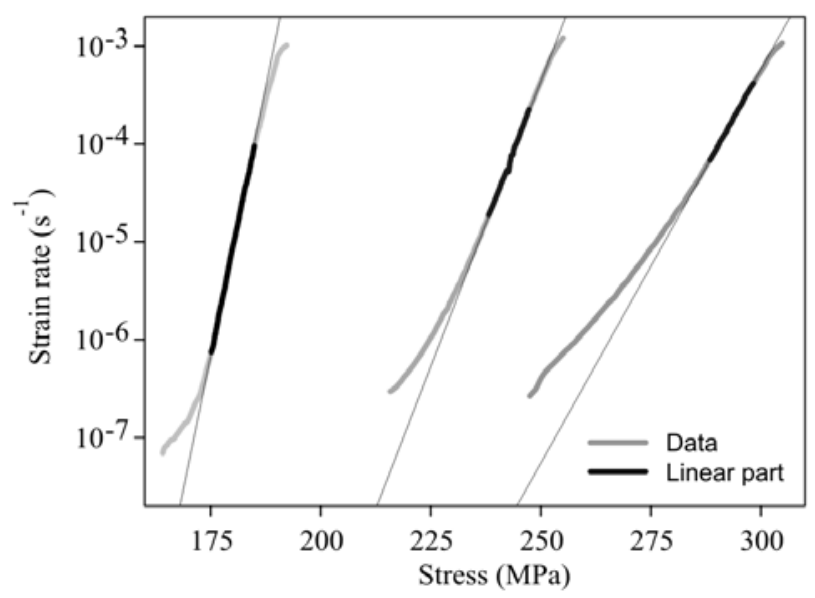

Fig. 3. Kinetic curves calculated from relaxation curves at room temperature.

etic curves. The SR tests started at the following flow stress/true strain: $193 \mathrm{MPa} / 0.016,255 \mathrm{MPa} / 0.06$, and $305 \mathrm{MPa} / 0.15$. A linear part of the plot occurs after a short period of about $1 \mathrm{~s}$; a short transition is observed. It is obvious that the slope of the plot decreases with increasing initial stress, i.e. at the stress at the beginning of the SR test. At longer time - at a lower plastic strain rate - departures from the linearity are observed. This indicates a process leading to dynamic recovery.

Some examples of the stress variation with the plastic strain rate at 100 and $200{ }^{\circ} \mathrm{C}$ (for lower and higher strains) are shown in Fig. 4. It is obvious that the shape of the curves is complex. It is difficult to find a longer linear part in the plots. A substantial contribution to dynamic recovery may be taken into account. Similar stress variations with the plastic strain rate were observed at 250 and $300^{\circ} \mathrm{C}$.

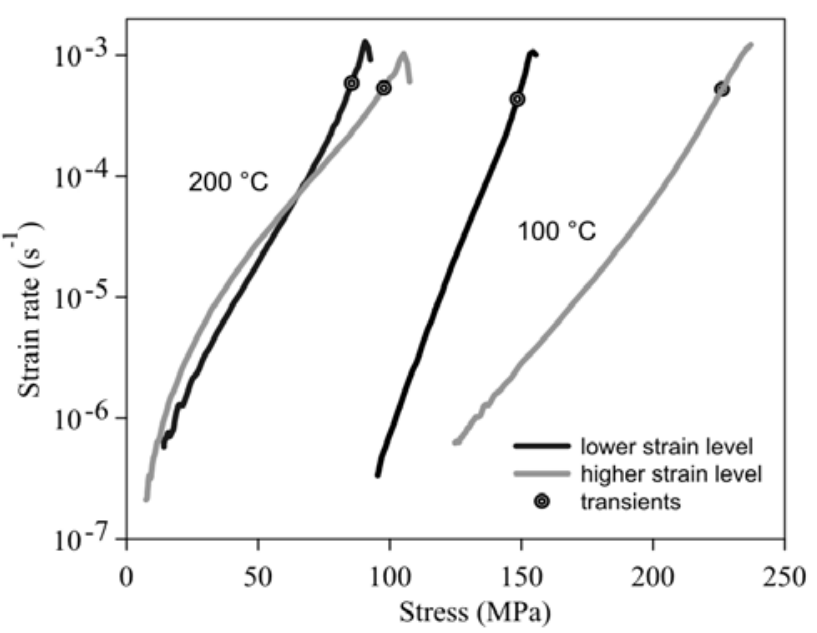

Fig. 4. Examples of kinetic curves determined from relaxation curves obtained at 100 and $200^{\circ} \mathrm{C}$ at lower and higher strain levels.

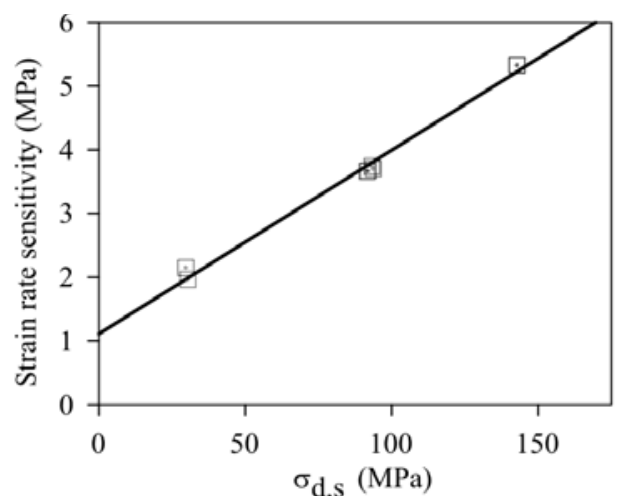

Fig. 5. Strain rate sensitivity of the flow stress, $M=M_{\mathrm{f}}+$ $k T / V$, at room temperature.

\section{Discussion}

The flow stress in polycrystalline magnesium alloys deformed by dislocation glide can be divided into three components:

$$
\sigma=\sigma_{\mathrm{d}}(\dot{\varepsilon}, T, S)+\sigma_{\mathrm{f}}(\dot{\varepsilon}, T)+\sigma_{\mathrm{n}}
$$

where $\sigma_{\mathrm{d}}$ is the dislocation component (flow resistance) due to interaction between moving dislocations and dislocation type obstacles, the density of which is usually varying with strain and enters the structure parameter $S, T$ is testing temperature. The friction stress, $\sigma_{\mathrm{f}}$, represents the resistance to dislocation motion due to solute atoms. It is also thermally activated but independent on straining. The stress component, $\sigma_{\mathrm{n}}$, is due to non-dislocation obstacles as grain boundaries, precipitates, dispersion particles.

The relation between the stress and plastic strain 
rate variation during stress relaxation can be expressed by the following equation:

$$
(1+\kappa h) \delta \sigma=\left\{\left(\partial \sigma_{\mathrm{d}} / \partial \ln \dot{\varepsilon}\right)+\left(\partial \sigma_{\mathrm{f}} / \partial \ln \dot{\varepsilon}\right)\right\} \delta \ln \dot{\varepsilon}\left(+\delta \sigma_{\mathrm{n}}\right),
$$

where $h$ is the strain hardening rate during SR and since $\kappa \approx 1 / 11.4 \mathrm{GPa}^{-1}$, the term $\kappa h$ can be omitted against 1 . We restrict ourselves to the linear part in plots in Fig. 3. Then $\partial \sigma_{\mathrm{n}}=0$; no change in non-dislocation obstacles occurs. The strain rate sensitivity (SRS) of the friction stress $M_{\mathrm{f}}=\left(\partial \sigma_{\mathrm{f}} / \partial \ln \dot{\varepsilon}\right)$ depends on the solute concentration as has been reported by Lukáč [15] who determined the concentration dependence of the activation volume of $\mathrm{Mg}-\mathrm{Cd}$ single crystal. $M_{\mathrm{f}}$ is independent of strain whereas the SRS of the dislocation stress $M_{\mathrm{d}}=\left(\partial \sigma_{\mathrm{d}} / \partial \ln \varepsilon \dot{\varepsilon}\right)$ depends on the initial stress of the relaxation, i.e. on the dislocation structure.

Combining Eqs. (2b), (3) and (4) gives the following relation for the dislocation stress:

$$
\begin{gathered}
\sigma_{\mathrm{d}}=\left(\Delta G_{0} / V\right)\left[1-\left(k T / \Delta G_{0}\right) \ln \left(\dot{\varepsilon}_{0} / \dot{\varepsilon}\right)\right]= \\
=\sigma_{\mathrm{d} 0}-\left[(k T / V) \ln \left(\dot{\varepsilon}_{0} / \dot{\varepsilon}\right)\right],
\end{gathered}
$$

where $\sigma_{\mathrm{d} 0}$ is the maximum stress at $0 \mathrm{~K}$; it is also called the mechanical maximum (the mechanical threshold) of the dislocation stress $[11,16]$. From the slope of the linear part of each plot in Fig. 3 one can estimate the strain rate sensitivity of the total stress $M$ $=\partial \sigma / \partial \ln \dot{\varepsilon}=M_{\mathrm{f}}+M_{\mathrm{d}}$. This quantity increases with increasing initial stress at the beginning $\mathrm{SR} \sigma_{\mathrm{s}}$ as mentioned above. The dislocation stress at the beginning $\mathrm{SR}, \sigma_{\mathrm{d}, \mathrm{s}}$, can be, to a first approximation, estimated as

$$
\sigma_{\mathrm{d}, \mathrm{s}} \approx \sigma_{\mathrm{s}}-\sigma_{0.2}
$$

where $\sigma_{0.2}$ is the yield strength. The strain rate sensitivity against the dislocation stress is plotted in Fig. 5 . This dependence - in the literature called the CottrellStokes law or the Haasen plot [17] has a slope of 0.0285 and a positive intercept $M_{\mathrm{f}} \approx 1.13 \mathrm{MPa}$. It is clear that the activation volume $V=k T / M$, (Eqs. (4) and (7)), decreases with increasing stress (strain) as has been reported for $\mathrm{Mg}-\mathrm{Cd}$ single crystals [15] and $\mathrm{Mg}$ -5Al-1Sr (wt.\%) polycrystalline alloy [4]. It should be mentioned that in this type of SR tests the apparent activation volume and not the physical activation volume was determined [10]. The values of the activation volume estimated for AZ31 magnesium alloy sheets (for instance $V / b^{3}=61$ at $\sigma=193 \mathrm{MPa}$ ) are close to those determined for as cast AJ51, AJ91 and AX41 alloys $[4,18]$.

The experimental results for samples deformed at temperatures above $100^{\circ} \mathrm{C}$ imply that the activation volume using an SR test can be determined only from data recorded at the very beginning of the test if the specimen is allowed to relax for a short time, about 200-300 s. These short duration SR were used for cast magnesium alloys as AZ31 and AJ51 [4, 5].

It was observed that at elevated temperatures the stress drop is higher than corresponding to Eq. (1), and the drop increases with increasing temperature. This indicates that during relaxation a process leading to softening (recovery) occurs. The activity of non-basal slip systems, e.g. double prismatic slip, may play a significant role in both hardening and softening processes. Screw components of $\langle a\rangle$ dislocations may move to the parallel slip planes by double cross slip through prismatic planes $[19,20]$. These phenomena will be analyzed in a future study.

\section{Conclusions}

The stress relaxation test is an effective method to study the thermally activated processes during deformation. At higher temperatures, the data for a short duration time (about $300 \mathrm{~s}$ ) should be used for determination of the activation volume. The tests with a long relaxation time at higher temperatures indicate a mechanism leading to dynamic recovery. Further investigations are required to clarify the effect of temperature and relaxation time on recovery mechanisms in AZ31 magnesium alloy sheets.

\section{Acknowledgement}

The authors gratefully acknowledge the financial support from the Czech Science Foundation under Grant 15$-11879 \mathrm{~S}$

\section{References}

[1] Feltham, P.: Phys. Stat. Sol., 3, 1963, p. 1340. doi:10.1002/pssb.19630030805

[2] Trojanová, Z., Drozd, Z., Lukáč, P., Máthis, K., Ferkel, H., Riehemann, W.: Scripta Mater., 42, 2000, p. 1095. doi:10.1016/S1359-6462(00)00342-0

[3] Trojanová, Z., Lukáč, P., Kainer, K. U.: Adv. Eng. Mater., 9, 2007, p. 370. doi:10.1002/adem.200700018

[4] Trojanová, Z., Lukáč, P.: Int. J. Mat. Res., 100, 2009, p. 270. doi:10.3139/146.110054

[5] Trojanová, Z., Balík, J., Lukáč, P.: Kovove Mater., 49, 2011, p. 385.

[6] Geng, J., Nie, J. F.: Scripta Mater., 100, 2015, p. 78. doi:10.1016/j.scriptamat.2014.12.018

[7] Wang, Y., Liu, Y., Wang, J. T.: Mater. Sci. Eng. A, 635, 2015, p. 86. doi:10.1016/j.msea.2015.03.054

[8] Li, J. C. M.: Canad. J. Appl. Phys., 45, 1967, p. 493. doi:10.1139/p67-043

[9] Dotsenko, V. I.: Phys. Stat. Sol. (b), 93, 1970, p. 11. doi:10.1002/pssb.2220930102 
[10] Caillard, D., Martin, J. L.: Thermally Activated Mechanisms in Crystal Plasticity. Oxford, Cambridge Univ. Press 2003.

[11] Kocks, U. K., Argon, A. S., Ashby, M. F.: Prog. Mater. Sci., 19, 1975, p. 1. doi:10.1016/0079-6425(75)90005-5

[12] Cáceres, C. H., Lukáč, P.: Phil. Mag., 88, 2008, p. 977. doi:10.1080/14786430801968611

[13] Balík, J., Lukáč, P., Bohlen, J., Kainer, K. U.: Kovove Mater., 45, 2007, p. 135.

[14] Balík, J., Lukáč, P., Drozd, Z., Kužel, R.: Int. J. Mat. Res., 100, 2009, p. 322. doi:10.3139/146.110042
[15] Lukáč, P.: Kovove Mater., 20, 1982, p. 372.

[16] Kocks, U. F., Mecking, H.: Prog. Mater. Sci., 48, 2003, p. 171. doi:10.1016/S0079-6425(02)00003-8

[17] Picu, R. C., Li, R.: Mater. Sci. Eng. A, 527, 2010, p. 5303. doi:10.1016/j.msea.2010.04.093

[18] Trojanová, Z., Lukáč, P., Száraz, Z.: Kovove Mater., 45, 2007, p. 283.

[19] Couret, A., Caillard, D.: Acta Metall., 33, 1985, p. 1447. doi:10.1016/0001-6160(85)90045-8

[20] Couret, A., Caillard, D.: Acta Metall., 33, 1985, p. 1455. doi:10.1016/0001-6160(85)90046-X 